\title{
Case of Advanced Recurrent Glioblastoma Successfully Treated with Monoterpene Perillyl Alcohol by Intranasal Administration
}

\author{
—-Intranasal POH Promotes Long-Term Survival of Recurrent Glioma
}

\author{
Clovis O. Da Fonseca ${ }^{1}$, Raphael M. Teixeira ${ }^{2}$, Ricardo Ramina ${ }^{3}$, Giovana Kovaleski ${ }^{4}$, \\ Júlio Thome Silva ${ }^{1}$, Janaína Nagel $^{5}$, Thereza Quirico-Santos ${ }^{2}$
}

\footnotetext{
${ }^{1}$ Department of General and Specialized Surgery; Unit of Clinical Research, Fluminense Federal University, RJ, Brazil; ${ }^{2}$ Institute of Biology, Fluminense Federal University; ${ }^{3}$ Institute of Neurology of Curitiba, Paraná; ${ }^{4}$ Medical Oncology Service, Rio de Janeiro; ${ }^{5}$ Getulio Vargas Estate Hospital, Rio de Janeiro.

Email: clovis.orlando@uol.com.br, tquirico@vm.uff.br
}

Received November $1^{\text {st }}$, 2010; revised November $15^{\text {th }}$, 2010; accepted November $17^{\text {th }}, 2010$.

\begin{abstract}
We report a case of recurrent glioblastoma (GBM) successfully treated with the Ras inhibitor monoterpene perillyl alcohol by intranasal administration. A 37-years-old white woman had been previously submitted to three neurosurgical procedures, in June 2000 for radical tumor excision of grade II astrocytoma; in July 2003 for first recurrence of type IV glioma and in August 2004 for GBM recurrence. After last surgery, patient started a new cycle of chemotherapy but was refractory to treatment, presented clinical adverse effects and resonance image scan showed no reduction of tumoral lesion. Patient was then considered out of therapeutic possibilities and indicated for supportive treatment. On March 2005 patient joined Phase I/II clinical trial for assess the efficacy of the monoterpene POH, a Ras inhibitor. $\mathrm{POH}$ was administered by intranasal route four times a day (268 $\mathrm{mg}$ daily) as single chemotherapy agent. Image scans performed 3 and 5 years later revealed marked reduction of enhancing lesion. This illustrative case demonstrates that intranasal administration of the monoterpene $\mathrm{POH}$ as a single agent was an effective therapeutic strategy capable to sustain long-term regression of recurrent glioma without clinical and laboratory toxicity.
\end{abstract}

Keywords: Glioblastoma, Long-Term Survival, Perillyl Alcohol, Intranasal Administration

\section{Introduction}

Prognosis for patients with recurrent glioblastoma (GBM) is dismal with a median survival of 3 - 6 months [1]. Factors that contribute to GBM recurrence include its infiltrative nature in the brain tissue which limits the effectiveness of complete surgical resection; presence of residual hypoxic tumor cells that are relatively resistant to radio and chemotherapy [2] and the blood-brain barrier (BBB) that restrains access of systemically administered therapy [3].

Current therapeutic strategies for patients with brain tumors are based on histological analysis which is insufficient for classification and grading of gliomas considering the molecular heterogeneity. Histological features vary from large ischemic and necrotic areas with intense angiogenesis frequently observed in primary glioblas- toma, to a minor fraction presenting foci of oligodendroglioma-like tumor cell differentiation [4-6] GBM may develop de novo (primary GBM) or through progression from low-grade astrocytoma (secondary GBM) indicating that it has evolved through different genetic pathways and probably differ in prognosis and response to therapy [5,7] Main genetic alterations of primary GBM include loss of heterozygosity 10q (70\% of cases), EGFR amplification (36\%), p16INK4a deletion (31\%), and PTEN mutations (25\%). In the pathway to secondary glioblastoma, TP53 mutations at CpG sites are the most frequent and earliest detectable genetic alteration already present in $60 \%$ of precursor low-grade astrocytoma. Yet, loss of heterozygosity $10 \mathrm{q} 25$-qter $(70 \%)$ is a frequent alteration common to both primary and secondary glioblastoma [7].

Activation of phosphatidylinositol-3-kinase (P13K/Akt) and Ras/MAPK pathways contributes to the malignant 
phenotype by disrupting cell cycle, increasing cell migration, enhancing cell survival and promoting angiogenesis [8-10]. Ras proteins, a family of small guanine nucleotide binding proteins involved in cellular signal transduction undergo several postranslational modifications that facilitate its attachment to the inner surface of the plasma membrane [11]. The first and most critical Ras modification to the biological active form is the addition of a farnesyl isoprenoid moiety in a reaction catalyzed by the enzyme farnesyltransferase (FTase) [12]. Anti-Ras pharmacyologic inhibitors under clinical evaluation include drugs able to prevent 1) association with the plasma membrane (prenylation and post prenylation inhibitors), 2) downstream signaling (kinase inhibitors) and 3) the upstream pathway (kinase inhibitor and monoclonal antibody) $[12,13]$. Perillyl alcohol $(\mathrm{POH})$ a dietary monoterpene found in essential oils of citrus peel, mints, celery seeds and several vegetables, presents in vitro and in vivo inhibitory effect upon cell cycle and survival pathways leading to apoptosis of transformed cells [14]. The chemopreventive activity of $\mathrm{POH}$ is partly related to inhibition of FTase enzyme and oxidative stress responses which ultimately halt tumor cell proliferation, and could be a useful therapeutic approach for patients with recurrent malignant glioma [15-17].

The efficacy of any anti-cancer drug greatly depends that therapeutic concentrations reaches the tumor site, a situation that is difficult to overcome concerning brain tumors due to the presence of the blood-brain barrier that restrains free drug access into the central nervous system [18]. However, the intranasal route provides a practical, noninvasive method for transportation of lipophilic and apolar drugs straight to the central nervous system [19]. In this context, we have been conducting a Phase I/II clinical trial to assess the efficacy of the monoterpene $\mathrm{POH}$ administered by intranasal route in patients with recurrent GBM [20,21]. This report aimed to document a case of recurrent GBM successfully treated with intranasal perillyl alcohol administration as single chemotherapeutic agent without any evidence of adverse effects for more than 5 years.

\section{Case Presentation}

The present study was approved by the University Hospital ethics committee and the Brazilian Ministry of Health (CONEP 9681 no. 124 25000.009267/2004-25). Before inclusion in the protocol, patient and her surrogates gave a written informed consent. The study was carried out in the Antonio Pedro Hospital Medical School of the Fluminense Federal University between 2005 and 2010 for a Phase I/II clinical trial to assess the therapeutic efficacy of $268 \mathrm{mg}$ POH daily administration by intranasal route.

A 37-years-old twelve-months pregnant white woman with no significant past medical record and without family history of brain tumor or neurological disorder had a seizure while sleeping at age 28 in October 1999. A brain magnetic resonance (MRI) scan revealed a regular space-occupying lesion in the right temporal lobe that enhanced with gadolinium. Patient underwent a right temporal craniotomy with radical resection of the primary brain tumor which was also confirmed by MRI scan performed after surgery. Histological analyzes confirmed diagnosis of grade II astrocytoma. Patient received medications for controlling seizures and remained asymptomatic until July 2003 when presented dizziness and painful headache. A new brain MRI revealed presence of right temporal mass with intense surrounding vasogenic edema. Patient promptly (August 2003) underwent a right temporal craniotomy with total tumor resection that was also confirmed by MRI. Histological analysis carried out by three different pathologists confirmed diagnosis of glioblastoma multiforme (GBM grade IV). Patient then started radiation therapy (59.4 Gy total) concomitant with chemotherapy schedule of 28-day cycle temozolomide $\left(150-200 \mathrm{mg} / \mathrm{m}^{2} /\right.$ day $)$ during 5 days. Six months after the end of such treatment (July 2004), patient developed persistent and painful headache accompanied by mild left-side hemiparesis. MRI revealed a lesion involving right temporal and parietal lobes with compression of the right ventricle. A craniotomy was performed in August 2004 but 3 months later the patient presented sustained left-side weakness, hemiparesis, abnormalities of gait and coordination. A new brain MRI revealed a right temporal mass with surrounding vasogenic edema (Figures 1(a), (c)) and characteristic histological alterations (necrosis, palisade cells, marked nuclear atypia and abnormal mitoses) characteristic of glioblastoma multiforme (Figure 2).

Patient started additional cycle of temozolomide schedule but treatment was withdrawn in February 2005 because patient was refractory to treatment, and presented clinical adverse effects (nausea, vomiting, thrombocytopenia) with resonance image scan showing no reduction of the tumoral lesion. The patient was then considered out of therapeutic possibilities and indicated for supportive (palliative) treatment. At this moment (August 2008), the patient was accepted to join Phase I/II protocol of intranasal administration of $268 \mathrm{mg} \mathrm{POH}$ daily as single chemotherapy. Intranasal $\mathrm{POH}$ treatment caused marked reduction of tumor size (Figures 1(b), (d)), great improvement of clinical condition and halt disease progression.

Brain MRI done in October 2009 still revealed irregular 


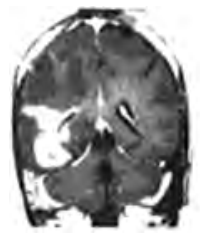

(a)

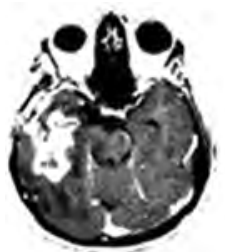

(d)

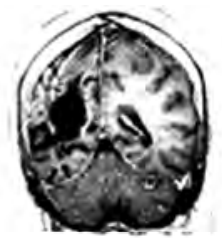

(b)

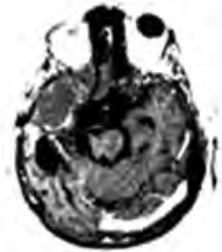

(e)

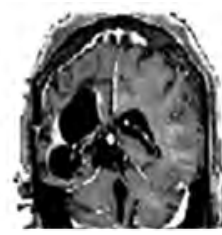

(c)

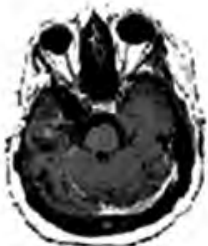

(f)

Figure 1. MRI brain image of recurrent GBM before and after $\mathrm{POH}$ intranasal administration. Note the decrease in tumor size between the initial MRI ((a); (d)) at the time of inclusion in POH protocol, images obtained in August 2008 ((b); (e)) and performed after 4 years and 9 months ((c) and (f)) of daily treatment with $\mathrm{POH}$ by intranasal route.

cystic lesions in the right cerebral hemisphere with area of contrast impregnation, loss of volumetric white matter, compensatory dilation of lateral and third ventricles with deviation of midline structures to the right side secondary to retractable effect. It was also observed reduced volume in the right cerebral peduncle secondary to Wallerian degeneration (Figures 3(a), (b)).

Patient remained in good health for more than 5 years (October 2010) and fully recovered from clinical neurological symptoms. Recent data from clinical laboratory analysis (Table 1) showed parameters within normal range values and also normal chest X-ray performed regularly at six monthly intervals since 2003 (data not shown). At the present moment patient still takes antiseizure medications but no steroidal drugs, and during all this period (more than 5 years) it was not observed any sign of toxicity or adverse effects caused by $\mathrm{POH}$ intranasal treatment.

\section{Discussion}

Only a subset of patients with newly diagnosed GBM exhibits a response to standard therapy [22]. Clinical failure of treatment for brain tumors may be largely attributed to a lack of appropriate brain drug delivery in a safe and effective manner [18]. Herein we report a case of a patient with recurrent GBM that had been successfully treated with intranasal administration of the monoterpene $\mathrm{POH}$ as single agent for more than five years without any side effects. As a result of intranasal $\mathrm{POH}$ treatment, the patient showed regression of tumor size

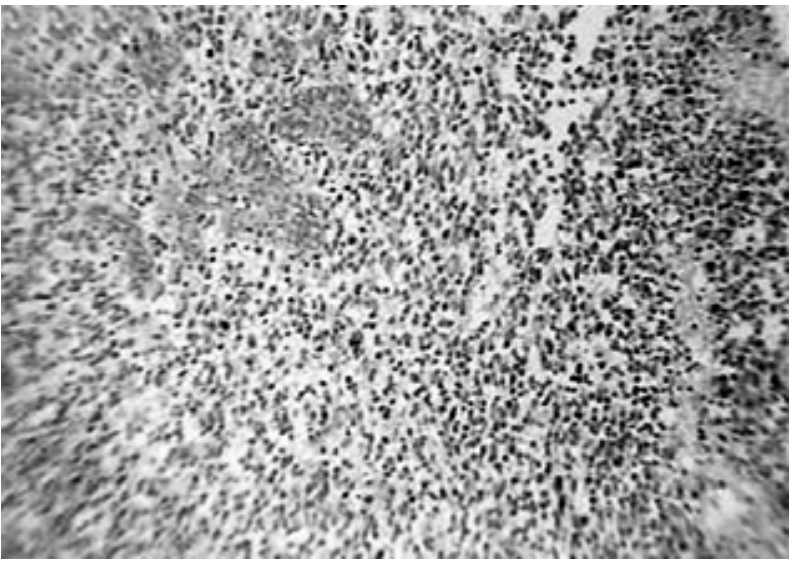

(a)

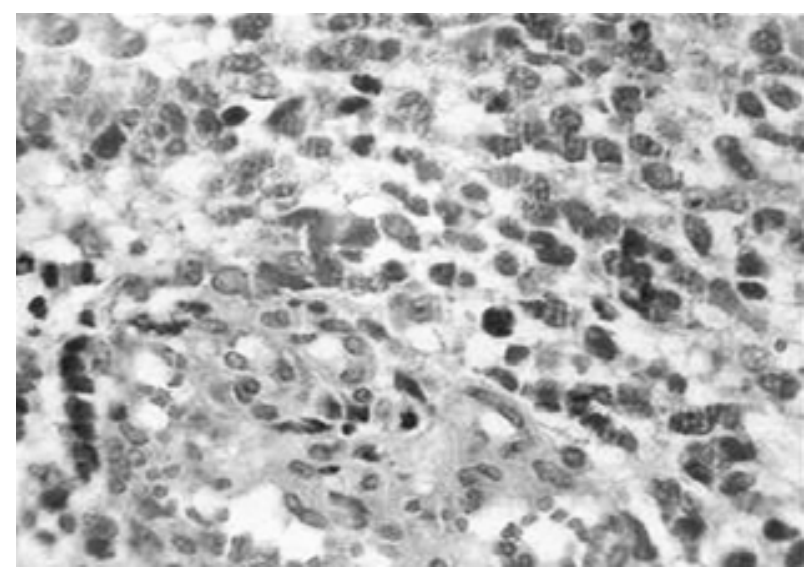

(b)

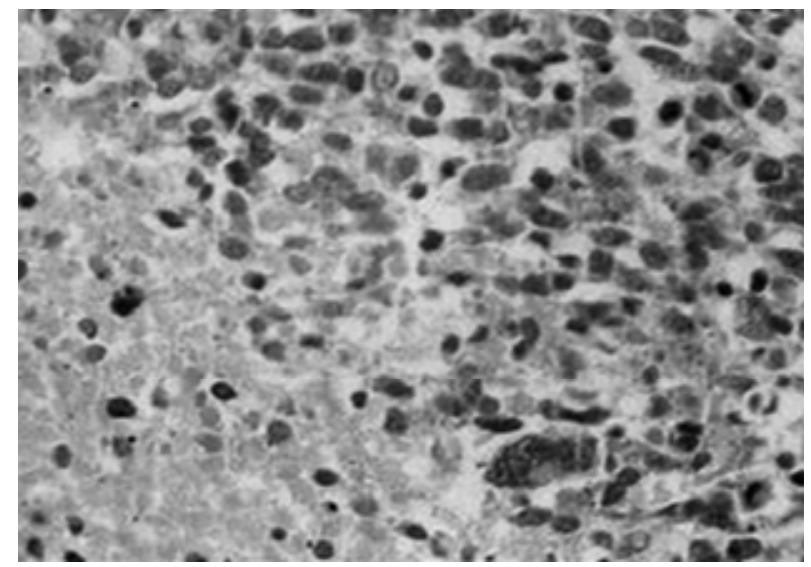

(c)

Figure 2. Histological characteristics of the tumor. Hematoxylin-eosin staining of paraffin-embedded brain tissue carried out in August 2003, showing characteristic features of glioblastoma. (a) Dense cellularity and microvascular proliferation. (b) microvascular proliferation with zone of coagulative necrosis lined by palisading tumor cell. (c) $100 x$ (a); 400x (b; c). 


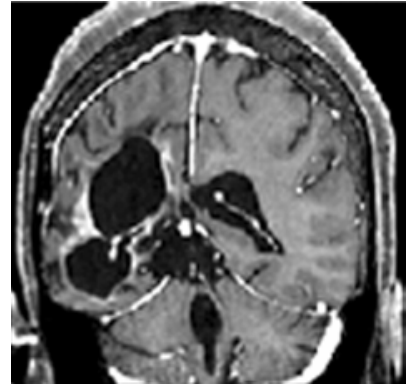

(a)

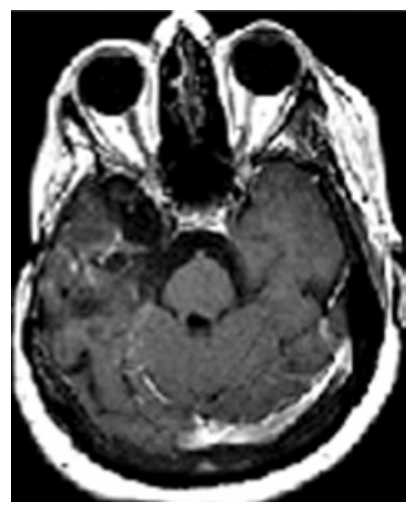

(b)

Figure 3. MRI brain image of recurrent GBM after 4 years treatment with $\mathrm{POH}$ by intranasal administration. Presence of irregular cystic lesions in the right cerebral hemisphere with area of contrast impregnation, compensatory dilation of lateral and third ventricles with deviation of midline structures and reduced volume in the right cerebral peduncle secondary to Wallerian degeneration.

and had a good prognosis despite dismal prognosis reported for GBM recurrence, that often presents a median survival of 3 - 6 months [1].

A prospective study carried out by our group [20] indicates that patients with recurrent secondary GBM have longer overall survival than those with primary recurrent GBM. Primary and secondary GBMs constitute distinct disease subtypes developing through different genetic alterations and signaling pathways [7,22]. Recently a microarray data predictive of poor patient outcome refractory to standard treatment, associate a panel of gene expression with mesenchymal differentiation and angiogenesis activation [22]. The EGFR-mediated signal transduction results in activation of a number of downstream pathways including p21-Ras-MAPK that inhibits apoptosis but activates proliferation and angiogenesis. Considering that activation of p21-Ras signal transduction is a characteristic event of a highly proliferative glioma, strategies inhibiting Ras prenylation could be used as an efficient adjuvant therapy. Data showing that
Table 1. Recent laboratory data of recurrent GBM after 5 years treatment with $\mathrm{POH}$ by intranasal administration.

\begin{tabular}{lccc}
\hline WBC & $7980 / \mathrm{mm}^{3}$ & LDH & $248 \mathrm{U} / \mathrm{L}$ \\
Neutrophil & $67 \%$ & Uric Acid & $2.9 \mathrm{mg} / \mathrm{dL}$ \\
Eosinophil & $9 \%$ & Folic acid & $8 \mathrm{ng} / \mathrm{mL}$ \\
Monocyte & $11 \%$ & & \\
Lymphocyte & $13 \%$ & $\mathrm{Na}^{+}$ & $139 \mathrm{mEq} / \mathrm{l}$ \\
RBC & $4.7 \mathrm{million} / \mathrm{mm}^{3}$ & $\mathrm{~K}^{+}$ & $4.1 \mathrm{mEq} / \mathrm{l}$ \\
Hb & $13.3 \mathrm{mg} / \mathrm{dl}$ & $\mathrm{Mg}^{+2}$ & $8.4 \mathrm{mg} / \mathrm{dL}$ \\
Ht & $40.50 \%$ & glucose $^{+2}$ & $98 \mathrm{mg} / \mathrm{dL}$ \\
Platelets & $350 \mathrm{000} / \mathrm{mm}^{3}$ & Urea & $9.1 \mathrm{mg} / \mathrm{dL}$ \\
CRP & $1.0 \mathrm{mg} / \mathrm{dL}$ & Creatinine & $0.9 \mathrm{mg} / \mathrm{dL}$ \\
Albumin & $4.3 \mathrm{mg} / \mathrm{dL}$ & Cholesterol & $283 \mathrm{mg} / \mathrm{dL}$ \\
Globulin & $2.9 \mathrm{mg} / \mathrm{dL}$ & Triglycerides & $99 \mathrm{mg} / \mathrm{dL}$ \\
T-Bilirubin & $0.33 \mathrm{mg} / \mathrm{dL}$ & HDL cholesterol & $50 \mathrm{mg} / \mathrm{dL}$ \\
D-Bilirubin & $0.12 \mathrm{mg} / \mathrm{dL}$ & VLDL cholesterol & $22 \mathrm{mg} / \mathrm{dL}$ \\
AFP & $60 \mathrm{U} / \mathrm{L}$ & AST & $16 \mathrm{IU} / \mathrm{L}$ \\
GGT & $30 \mathrm{U} / \mathrm{L}$ & ALT & $22 \mathrm{IU} / \mathrm{L}$ \\
\hline
\end{tabular}

$\mathrm{POH}$ inhibits prenylation of Ras and other proteins in many cell types [23] support the view that POH could also be effective against glioma cells. Indeed data from our group showed that POH induced apoptosis, inhibited angiogenesis and migration of glioma cell lines and from human tumor explants [17]. Likewise clinical studies showed that the intranasal formulation of $\mathrm{POH}$ prolonged overall survival and reduced tumor size of recurrent GBM $[20,24,25]$. We herein report a case of a patient with recurrent GBM that presents stable disease, without any clinical evidence of recurrence for more than 5 years of treatment exclusively with daily administration by intranasal route of $268 \mathrm{mg} \mathrm{POH}$, a Ras inhibitor. This therapeutic strategy is a noninvasive approach that can reduce systemic side effects, because overcome the need for hepatic processing often necessary for activation of most drugs administered by oral or intravenous route $[26,27]$. It is conceivable that a lipophilic agent like $\mathrm{POH}$ administered by intranasal route might also be used as a carrier transporting anti-neoplastic drugs straight into the central nervous system. The present study shows an efficient response of recurrent glioblastoma to intranasal administration of $\mathrm{POH}$, a safe and well-tolerated therapeutic regimen with clear antitumor activity. 


\section{Acknowledgments}

This study was supported by grants from FAPERJ (Fundação de Amparo a Pesquisa do Rio de Janeiro number E-26/111.425/2008) and CNPq (Brazilian Research Council number 470441/2008-9).

\section{REFERENCES}

[1] D. S. Kong, J. I. Lee, J. H. Kim, et al., "Phase II Trial of Low-Dose Continuous (Metronomic) Treatment of Temozolomide for Recurrent Glioblastoma," Neurology Oncology, Vol. 12, No. 3, 2010, pp. 289-296.

[2] J. E. Chang, D. Khuntia, H. I. Robins, et al., "Radiotherapy and Radiosensitizers in the Treatment of Glioblastoma Multiforme,” Clinical Advanced Hematology Oncology, Vol. 5, No. 11, 2007, pp. 894-902, 907-915.

[3] H. L. Liu, M. Y. Hua, P. Y. Chen, et al., "Blood-Brain Barrier Disruption with Focused Ultrasound Enhances Delivery of Chemotherapeutic Drugs for Glioblastoma Treatment,” Radiology, Vol. 255, No. 2, 2010, pp. 415425. doi:10.1148/radiol.10090699

[4] T. Nagasaka, M. Gunji, N. Hosokai, et al., "FISH 1p/19q Deletion/Imbalance for Molecular Subclassification of Glioblastoma,” Brain Tumor Pathology, Vol. 24, No. 1, 2007, pp. 1-5. doi:10.1007/s10014-006-0209-6

[5] B. Klink, B. Schlingelhof, M. Klink, et al., "Glioblastomas with Oligodendroglial Component-Common Origin of the Different Histological Parts and Genetic Subclassification,” Cell Oncology, May 25, 2010.

[6] T. Miyanaga, J. Hirato and Y. Nakazato, “Amplification of the Epidermal Growth Factor Receptor Gene in Glioblastoma: An Analysis of the Relationship between Genotype and Phenotype by CISH Method," Neuropathology, Vol. 28, No. 2, 2007, pp. 116-126. doi:10.1111/j.1440-1789.2007.00853.x

[7] H. Ohgaki and P. Kleihues, "Genetic Pathways to Primary and Secondary Glioblastoma," American Journal of Pathology, Vol. 170, No. 5, 2007, pp. 1445-1453. doi:10.2353/ajpath.2007.070011

[8] M. Kanamori, T. Kawaguchi, J. M. Nigro, et al., "Contribution of Notch Signaling Activation to Human Glioblastoma Multiforme,” Journal Neurosurg, Vol. 106, No. 3, 2007, pp. 417- 427. doi:10.3171/jns.2007.106.3.417

[9] C. B. Knobbe and G. Reifenberger, "Genetic Alterations and Aberrant Expression of Genes Related to the PhosPhatidyl-Inositol-3'-Kinase/protein Kinase B (Akt) Signal Transduction Pathway in Glioblastomas,” Brain Pathology, Vol. 13, No. 4, 2003, pp. 507-518. doi:10.1111/j.1750-3639.2003.tb00481.x

[10] R. Blum, I. Nakdimon, L. Goldberg, et al., "E2F1 Identified by Promoter and Biochemical Analysis as a Central Target of Glioblastoma Cell-Cycle Arrest in Response to Ras Inhibition," International Journal of Cancer, Vol. 119, No. 3, 2006, pp. 527-538. doi:10.1002/ijc.21735
[11] J. Guldenhaupt, Y. Adiguzel, J. Kuhlmann, et al., "Secondary Structure of Lipidated Ras Bound to a Lipid Bilayer,” FEBS Journal, Vol. 275, No. 23, 2008, pp. 5910-5918. doi:10.1111/j.1742-4658.2008.06720.x

[12] A. C. Berzat, D. C. Brady, J. J. Fiordalisi, et al., "Using Inhibitors of Prenylation to Block Localization and Transforming Activity," Methods in Enzymology, Vol. 407, 2006, pp. 575-597. doi:10.1016/S0076-6879(05)07046-1

[13] N. Saxena, S. S. Lahiri, S. Hambarde, et al., "RAS: Target for Cancer Therapy,” Cancer Investigation, Vol. 26, No. 9, November 2008, pp. 948-955. doi:10.1080/07357900802087275

[14] B. H. Chung, H. Y. Lee, J. S. Lee, et al., "Perillyl Alcohol Inhibits the Expression and Function of the Androgen Receptor in Human Prostate Cancer Cells,” Cancer Letter, Vol. 236, No. 2, May 18, 2006, pp. 222-228. doi:10.1016/j.canlet.2005.05.023

[15] S. C. Chaudhary, M. S. Alam, M. S. Siddiqui, et al., "Perillyl Alcohol Attenuates Ras-ERK Signaling to Inhibit Murine Skin Inflammation and Tumorigenesis," Chemical Biology Interact, Vol. 179, No. 2-3, 15, 2009, pp. 145-153. doi:10.1016/j.cbi.2008.12.016

[16] C. O. da Fonseca, J. A. Landeiro, S. S. Clark, et al., "Recent Advances in the Molecular Genetics of Malignant Gliomas Disclose Targets for Antitumor Agent Perillyl Alcohol,” Surgery Neurology, Vol. 65, Suppl. 1, 2006, pp. 2-9.

[17] J. Fernandes, C. O. da Fonseca, A. Teixeira, et al., "Perillyl Alcohol Induces Apoptosis in Human Glioblastoma Multiforme Cells,” Oncology Reports, Vol. 13, No. 5, 2005, pp. 943-947.

[18] M. M. Patel, B. R. Goyal, S. V. Bhadada, et al., "Getting into the Brain: Approaches to Enhance Brain Drug Delivery,” CNS Drugs, Vol. 23, No. 1, 2009, pp. 35-58. doi:10.2165/0023210-200923010-00003

[19] H. Wu, K. Hu and X. Jiang, "From Nose to Brain: Understanding Transport Capacity and Transport Rate of Drugs,” Expert Opinion on Drug Delivery, Vol. 5, 2008, pp. 1159-1168. doi:10.1517/17425247.5.10.1159

[20] C. da Fonseca, M. Simão, I. Lins, et al., "Efficacy of Monoterpene Perillyl Alcohol upon Survival Rate of Patients with Recurrent Glioblastoma," Journal of Cancer Research and Clinical Oncology, 2010, pp. 1-7.

[21] C. O. da Fonseca, R. Linden, D. Futuro, et al., "Ras Pathway Activation in Gliomas: A Strategic Target for Intranasal Administration of Perillyl Alcohol," Archivum Immunologiae et Therapia Experimentalis (Warsz), Vol. 56, No. 4, 2008, pp. 267-276. doi:10.1007/s00005-008-0027-0

[22] H. Colman, L. Zhang, E. P. Sulman, et al., "A Multigene Predictor of Outcome in Glioblastoma,” Neuro Oncology, Vol. 12, No. 1, 2010, pp. 49-57.

[23] S. S. Clark, L. Zhong, D. Filiault, et al., “Anti-Leukemia Effect of Perillyl Alcohol in Bcr/Abl-Transformed Cells Indirectly Inhibits Signaling through Mek in a Ras- and 
Raf-Independent Fashion,” Clinical Cancer Research, Vol. 9, No. 12, 2003, pp. 4494-4504.

[24] C. O. da Fonseca, G. Schwartsmann, J. Fischer, et al., "Preliminary Results from a Phase I/II Study of Perillyl Alcohol Intranasal Administration in Adults with Recurrent Malignant Gliomas,” Surgery Neurology, Vol. 70, No. 3, 2008, pp. 259-266. doi:10.1016/j.surneu.2007.07.040

[25] C. O. Da Fonseca, J. T. Silva, I. R. Lins, et al., "Correlation of Tumor Topography and Peritumoral Edema of Recurrent Malignant Gliomas with Therapeutic Response to Intranasal Administration of Perillyl Alcohol,” Investigational New Drugs, January 13, 2009.

[26] P. Kleihues and H. Ohgaki, "Primary and Secondary Glioblastomas: From Concept to Clinical Diagnosis," Neuro Oncology, Vol. 1, No. 1, January 1999, pp. 44-51.

[27] Y. Zhu, F. Guignard, D. Zhao, et al., "Early Inactivation of P53 Tumor Suppressor Gene Cooperating with NF1 Loss Induces Malignant Astrocytoma,” Cancer Cell, Vol. 8, No. 2, 2005, pp. 119-130. doi:10.1016/j.ccr.2005.07.004 